Document downloaded from:

http://hdl.handle.net/10251/148100

This paper must be cited as:

Morales, S.; Bernabeu-Sanz, A.; López-Mir, F.; Gonzalez, P.; Luna, L.; Naranjo Ornedo, V. (2017). BRAIM: A computer-aided diagnosis system for neurodegenerative diseases and brain lesion monitoring from volumetric analyses. Computer Methods and Programs in Biomedicine. 145:167-179. https://doi.org/10.1016/j.cmpb.2017.04.006

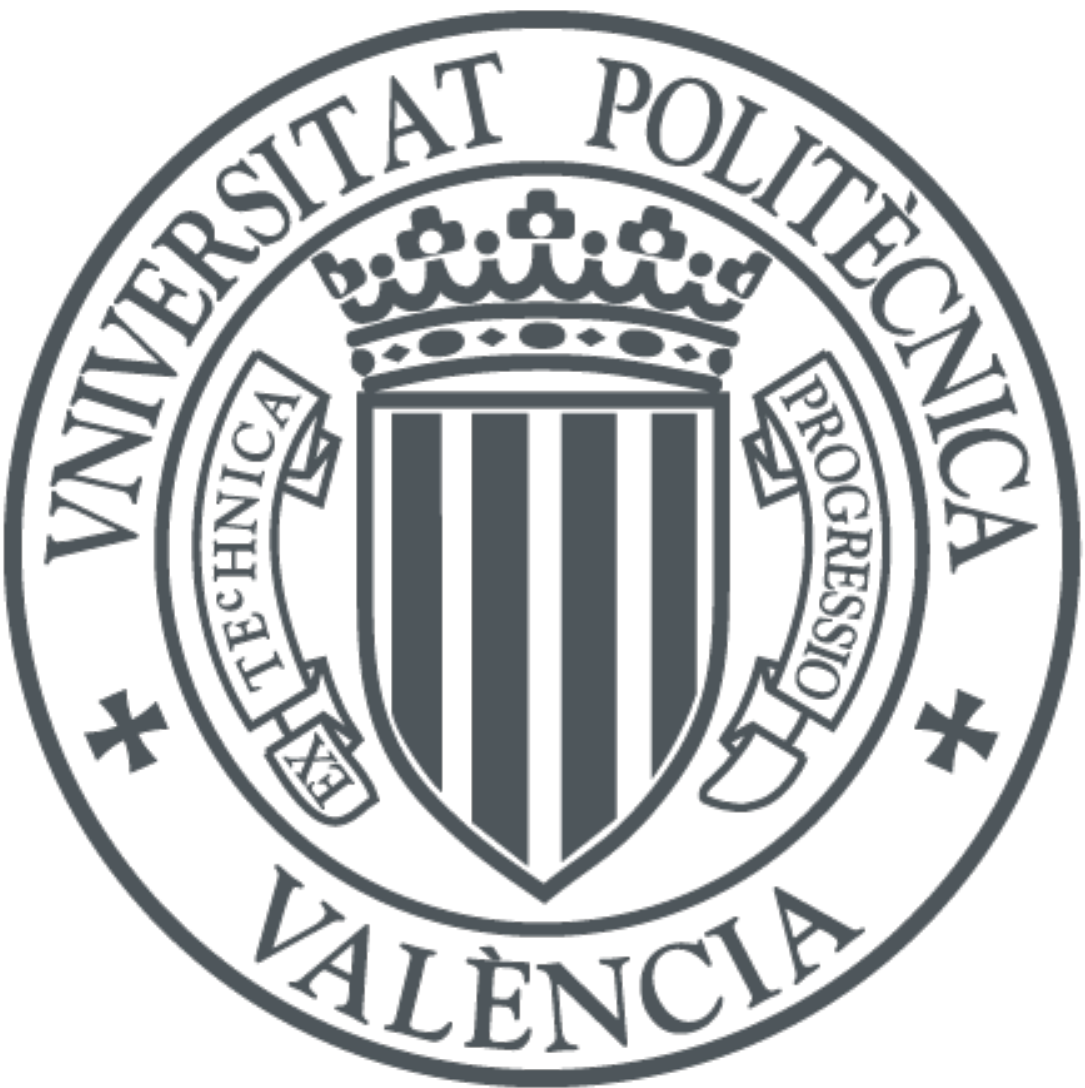

The final publication is available at

https://doi.org/10.1016/j.cmpb.2017.04.006

Copyright Elsevier

Additional Information 


\title{
BRAIM: A computer-aided diagnosis system for neurodegenerative diseases and brain lesion monitoring from volumetric analyses.
}

\author{
Sandra Morales ${ }^{\mathrm{a}, *}$, Angela Bernabeu-Sanz ${ }^{\mathrm{b}}$, Fernando López-Mir ${ }^{\mathrm{a}}$, Pablo \\ González $^{\mathrm{a}}$, Luis Luna ${ }^{\mathrm{b}}$, Valery Naranjo ${ }^{\mathrm{a}}$ \\ ${ }^{a}$ Instituto de Investigación e Innovación en Bioingeniería, I3B, Universitat Politècnica de \\ València, Camino de Vera s/n, 46022, Valencia, Spain \\ ${ }^{b}$ Inscanner S.L, Magnetic Resonance Department, Alicante, Spain
}

\begin{abstract}
Background and Objective: This paper presents BRAIM, a computer-aided diagnosis (CAD) system to help clinicians in diagnosing and treatment monitoring of brain diseases from magnetic resonance image processing. BRAIM can be used for early diagnosis of neurodegenerative diseases such as Parkinson, Alzheimer or Multiple Sclerosis and also for brain lesion diagnosis and monitoring. Methods: The developed CAD system includes different user-friendly tools for segmenting and determining whole brain and brain structure volumes in an easy and accurate way. Specifically, three types of measurements can be performed: (1) total volume of white, gray matter and cerebrospinal fluid; (2) brain structure volumes (volume of putamen, thalamus, hippocampus and caudate nucleus); and (3) brain lesion volumes. Results: As a proof of concept, some study cases were analyzed with the presented system achieving promising results. In addition to be used to quantify treatment effectiveness in patients with brain lesions, it was demonstrated that BRAIM is able to classify a subject according to the brain volume measurements using as reference a healthy control database created for this purpose. Conclusions: The CAD system presented in this paper simplifies the daily work of clinicians and provides them
\end{abstract}

\footnotetext{
* Corresponding author

Email address: sanmomar@i3b.upv.es (Sandra Morales)
} 
with objective and quantitative volume data for prospective and retrospective analyses.

Keywords: Magnetic Resonance Imaging; Volumetric analysis; CAD; Brain segmentation; Brain lesions;

\section{Introduction}

Neurological disorders are one of the most common health problems of the population that negatively affect their individual autonomy and well-being. Besides the physiological aging and natural cognitive decline, numerous diseases such as Parkinson disease, Multiple Sclerosis, and many common processes in the adult population (i.e. infections, neoplasms, systemic diseases, drug consumption, etc.) can adversely affect cognitive functions both acute or chronically $[1,2]$. Regardless etiology, in all cases an early diagnosis is crucial to proceed with appropriate therapeutic measures in order to improve patients quality of life and/or moderate the development of clinical complications $[3,4,5]$.

In the clinical setting, recent studies have highlighted the relevance of performing volumetric measurements instead of the classical approach of qualitative analyses. For example, in neurodegenerative disorders researchers have shown that early brain atrophy detection (which is not possible by visual inspection of the MR images) is extremely valuable for early diagnosis and better patient management $[6,7,8]$. In oncology, accurate volume tumor measurements are a critical indicator of disease progression and/or treatment efficacy. Particularly, when assessment methods use robust tumor volume measurements, the obtained information constitutes the most important predictor of patient outcome $[9,10,11]$.

However, and despite the proven clinical relevance of brain volumetric analyses, this technique is not usually performed in the clinical setting. The main reasons are that it is somewhat tedious and time-consuming, needs specialized personnel, a suitable software, and a specific Magnetic Resonance Imaging (MRI) protocol $[12,13]$. 
Additionally, and despite the fact that the process a priori seems quite simple, there are not adequate or user-friendly tools available for clinicians at the moment. There are many applications that help the process and are able to brain segmentation, but in most cases their use and installation are complex requiring the installation of many other data libraries as well as computation skills. Besides, most of the available software for brain segmentation are black boxes that provide automatic segmentation results not allowing any type of amend or adjustment if the results are not satisfactory. In general, this type of procedure works accurately in healthy brains but frequently fails in the presence of lesions, significant brain atrophy or anatomical variations. Therefore, in clinical studies it is very common that some pathological cases must be removed from the investigation despite the image acquisition was adequate; because the automatic segmentation provided by the software is not correct.

In this paper, we present a new computer-aided diagnosis (CAD) system for clinicians. BRAIM is a software tool capable to perform brain segmentation and robust volumetric analyses. It could be operated in a common PC with Windows operating system and does not require any specific computing skills to use it. It allows semi-automated and automated segmentation of the brain and its structures, as well as manual correction of the segmentation results in case they are not optimal. In addition, it is featured with an internal MRI database from healthy controls that can be continuously upgraded by the operator. Making use of this control database, the system is able to classify the patient status according to the segmentation results. As it will be shown in the next sections BRAIM CAD system offers many advantages for clinicians as it could be easily used in the clinical setting, it is fast, user-friendly and intuitive.

The paper is organized as follows: first, materials and methods are described. Secondly, a description in depth of the functionalities of the CAD system presented in the paper is included. Next section presents some results to demonstrate the software usefulness. Finally, discussion, conclusion and some future work areas are provided. 


\section{Materials and methods}

\subsection{Subjects}

In order to test and validate the software, several brain MRI studies were obtained. Candidate selection was performed as follows.

\subsubsection{Healthy subjects}

50 healthy subjects ( 25 male, 25 female; mean age $44.14 \pm 14.2$; range $22-70$ years) were included in the study and used to create a control database. In order to ensure a homogenous distribution of the data base results, ten subjects were included for each decade of age (5 males, 5 females). Subjects were recruited from the department of Neurology at the "Hospital Universitario de Alicante" by an experienced Neurologist. Suitability of participants as healthy controls, was ensured after clinical history and cognitive and physical examination. In addition each participant performed two cognitive test, specifically the SDMT (symbol digit modality test) [14] and the T@M memory test [15]. Both tests were used to rule out any cognitive problem that could negatively affect the investigation. Exclusion criteria included claustrophobia, metal implants, visual or neurological disease, epilepsy, drug abuse, psychiatric disease, head injury or any other conditions that could negatively affect the subject cognition.

\subsubsection{Patient selection}

Patients included in this paper as examples are part of a bigger study (BRAIM project, www.i3b.webs.upv.es/cvblab/en/braim-2/) and were selected by specialized clinicians from the departments of Neurology and Neurosurgery both located in the "Hospital Universitario de Alicante", Spain. In particular, four patients were selected to illustrate the BRAIM functioning:

- Patient A: a 47 year-old female patient diagnosed with Multiple Sclerosis subtype remitent-recidivant for eleven years. Neurological tests suggested mild cognitive impairment with SDMT score of 27 (normative value $\geq 42$ $[14,16,17])$ and T@M results of 41 (cut-off value in elderly population $>37[18,19])$. 
- Patient B: a 48 year-old female patient diagnosed with Multiple Sclerosis subtype remitent-recidivant for fourteen years. Neurological tests did not suggest cognitive impairment with SDMT score of 42 (normative value $\geq$ $42[14,16,17])$ and $\mathrm{T} @ \mathrm{M}$ results of 49 (cut-off value in elderly population $>37[18,19])$.

- Patient C: a 25 year-old female diagnosed with a pontine tumor. Biopsy was not performed because of the lesion location. Chemotherapy was administrated in order to control tumor growth.

- Patient D: a 27 year-old male diagnosed with brain astrocitoma III. The lesion affected both frontal lobes and corpus callosum. After surgery chemotherapy and radiotherapy were administrated to the patient in order to control the rest tumor growth.

The protocol was approved by the institutional review board and the "Agencia Española del Medicamento y Productos sanitarios" (www.aemps.gob.es). All participants were informed about the study and gave their written informed consent prior to entering the study.

\subsection{MRI protocol}

MRI studies were performed on a 3T Philips Achieva with a SENSE Neurovascular coil 16 elements provided by the manufacturer (Philips, The Netherlands). The MRI protocol consisted of a high-resolution T1-weighted turbo gradient-echo scan: 160 slices, $1 \mathrm{~mm}$ isotropic voxels, field of view $250 \times 250$ $\mathrm{mm}$, TR $13 \mathrm{~ms}$ and TE $7 \mathrm{~ms}$, Flip angle $8^{\circ}$ in axial plane. In the case of brain tumor, images were acquired after contrast injection (Gadovist, $0.1 \mathrm{mg} / \mathrm{kg}$ ).

After acquisition, all images were transferred to a workstation (Philips, The Netherlands) and checked by an experienced radiologist to ensure the absence of artifacts or any brain lesions that could negatively affect the study.

\subsection{Volumetric measurements and MRI analyses}

The MRI studies of the 50 healthy selected subjects were analyzed with BRAIM software. After skull striping, automated segmentation of white mat- 
ter, gray matter and CSF was performed. These automatic segmentation results were saved and semi-automatic segmentation was performed for both thalamus, hippocampus, caudate nucleus and putamen. Afterwards, volume of the segmented tissues and structures were computed and the results were added progressively to the database taking into account the age of the patient.

MRI of Multiple Sclerosis patients (Patient A and B) were also analyzed with BRAIM software following the same procedure as described previously and the obtained values were introduced in the classification system for patient comparison within de database.

In the case of brain tumor images (Patient $\mathrm{C}$ and $\mathrm{D}$ ), only the lesion was segmented by automatic procedures with BRAIM software and manual editing was performed afterwards if necessary.

\subsection{Statistical analyses}

All statistical analyses were performed using SPSS Version 19.0 (SPSS Inc., Chicago, IL). Results are expressed as mean \pm standard deviation. We used the Kolmogorov-Smirnoff test for determining the normal distribution of the data. Two different Paired Student t-tests were performed to compare volumetric data results: a t-test to compare interhemispheric volumetric data for basal ganglia, thalamus and hippocampus structures; a second t-test was performed to assess differences in white matter, gray matter and cerebrospinal fluid between sexes. A p value $<0.05$ was considered statistically significant.

\subsection{System requirements}

BRAIM software is a program written in $\mathrm{C} / \mathrm{C}++$ compiled for Windows

platforms. It was developed making use of several open-source libraries: Qt 4.8.1 (www.qt.io/) for graphical user interface creation; Insight Segmentation and Registration ToolKit (ITK 3.20, itk.org/) for image analysis; Visualization ToolKit (VTK 5.10, www.vtk.org/) for 3D rendering and representation; and Statistical Parametric Mapping (SPM12, www.fil.ion.ucl.ac.uk/spm/) for neuroimaging data analysis. SPM is a suite of MATLAB (The MathWorks, 
Inc.) functions that were compiled with MATLAB Compiler toolbox 7.16. The use of the functions belonging to these libraries is combined along with own algorithms.

BRAIM is available for Windows (XP/Vista/Windows 7/Windows 10). All the required libraries were embedded in the tool installer and do not require commercial licenses for research purposes. It requires less than $1.2 \mathrm{~GB}$ of free hard disk space for its installation (260 MB for BRAIM and 898 MB for MATLAB Compiler Runtime).

\section{CAD system description}

The goal of the CAD system presented in this paper is to analyze the volume of specific brain regions including structures and lesions from a 3D brain MRI. In Figure 1, the BRAIM architecture is depicted. The aspect of its graphical user interface can be observed in Figure 2. Note that tissue segmentation, i.e. white matter, gray matter and cerebrospinal fluid segmentation, and skullstripping are performed making use directly of SPM software package; structure segmentation is implemented by means of an interactive polygon tool designed in BRAIM; and lesions are segmented by own algorithms.

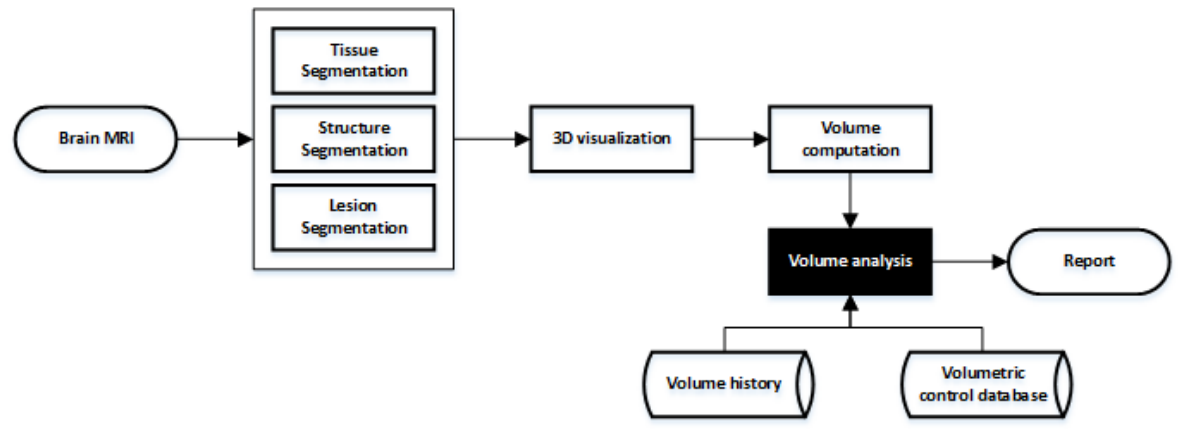

Figure 1: Schematic representation of BRAIM architecture.

BRAIM software is designed to be used in the daily work of clinicians, so it works on DICOM images. BRAIM is able to load and work not only MRI 


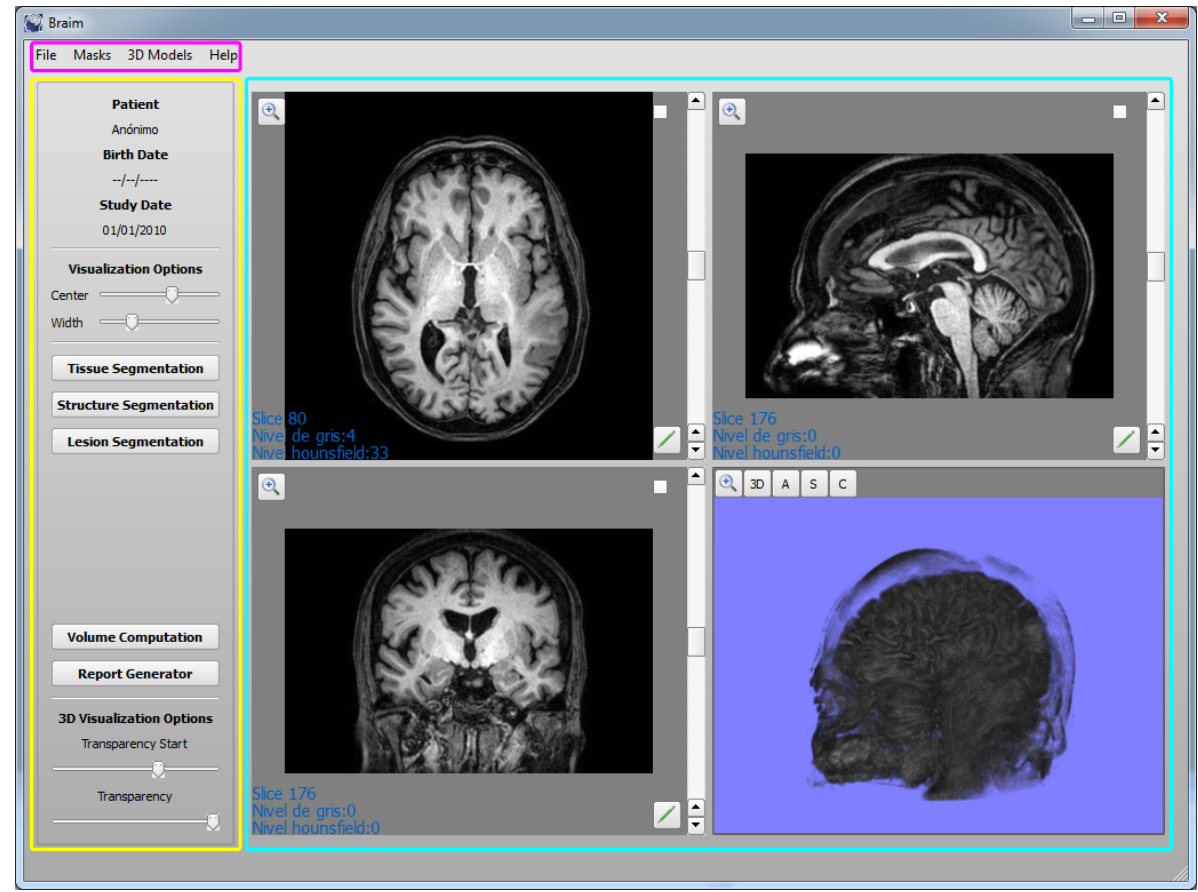

Figure 2: BRAIM graphical user interface. The main components are shown, the menu bar (magenta), visualization windows (cyan) and panels (yellow).

but also other medical image modalities as long as they are in DICOM format. However, as the main objective of this software is to study the brain in a highresolution detail as well as to perform accurate volumetric analyses, MRI is the proper modality for this purpose; it has a great range of available soft tissue contrast, depicts anatomy in great detail and is sensitive and specific for abnormalities within the brain itself.

When a brain MRI is loaded, the anatomic study is represented in axial, sagittal and coronal view (Figure 2, right). The software loads the main plane (usually axial) and interpolates the other missing planes (usually coronal and sagittal). This allows to a better perception of the brain structures and/or lesions to be segmented. If it is needed, a $3 \mathrm{D}$ view of the brain can also be generated.

Later, the main actions of the software are enabled (Figure 2, left). Next, 
they will be described in depth. The main functions and libraries used for their implementation are detailed in Appendix A.

\subsection{Visualization options}

A Window/Level function can be applied, if it is needed, to enhance the image contrast and brightness and improve the visualization of the brain and its structures. This function applies a linear gray-scale transformation by means of a lookup table (LUT) specified by window (W) and level (L) parameters. These parameters define a specific range of gray level intensities to be highlighted. The gray level intensities outside the range are transformed to the minimum or maximum value of the range, as applicable.

\subsection{Brain tissue segmentation}

Total white matter (WM), gray matter (GM) and cerebrospinal fluid (CSF) are automatically segmented using SPM software package (SPM12, www.fil. ion.ucl.ac.uk/spm/). SPM defines the tissue types according to probability maps obtained through a modified Gaussian Mixture Model [20]. A Gaussian mixture model is a parametric probability density function represented as a weighted sum of Gaussian component densities.

A distribution can be modelled by a mixture of $K$ Gaussians (clusters) where each cluster $k$ is described by its number of voxels $\left(h_{k}\right)$, mean $\left(v_{k}\right)$ and variance $\left(c_{k}\right)$. In particular, SPM is based on a Bayesian formulation of the Gaussian mixture model with a prior probability image obtained by averaging an already segmented large number of brain images. Based on the prior probability of each voxel belonging to a specific tissue type, the Bayesian framework is used to get the posterior probability. Let $\mathbf{F}$ be an image of $I \times J$ voxels, the posterior probability $p_{i j k}$ that a voxel $(i, j)$ belongs to cluster $k$ given its intensity of $f_{i j}$ is defined as follows

$$
p_{i j k}=\frac{r_{i j k} s_{i j k}}{\sum_{l=1}^{K} r_{i j l} s_{i j l}} \text { over } i=1 \ldots I, j=1 \ldots J \text { and } k=1 \ldots K,
$$


where $r_{i j k}$ is the likelihood of a voxel in cluster $k$ having an intensity of $f_{i j}$, and $s_{i j k}$ is the a priori probability of voxel $(i, j)$ belonging in cluster $k$.

The likelihood function is obtained by evaluating the probability density functions for each voxel of each cluster:

$r_{i j k}=\frac{u_{i j}}{\left(2 \pi c_{k}\right)^{1 / 2}} \exp \left(\frac{-\left(f_{i j} u_{i j}-v_{k}\right)^{2}}{2 c_{k}}\right)$ over $i=1 \ldots I, j=1 \ldots J$ and $k=1 \ldots K$,

being $\mathbf{U}$ a modulation function that represents the intensity and noise associated with each voxel in the image by multiplication with a smooth scalar field and $u_{i j}$ the particular multiplicative correction at voxel $(i, j)$.

The prior $\left(s_{i j k}\right)$ is based on two factors: the number of voxels currently belonging to each cluster $\left(h_{k}\right)$, and the prior probability images derived from a large number of images previously segmented $\left(b_{i j k}\right)$ :

$s_{i j k}=\frac{h_{k} b_{i j k}}{\sum_{l=1}^{I} \sum_{m=1}^{J} b_{l m k}} \exp \left(\frac{-\left(f_{i j} u_{i j}-v_{k}\right)^{2}}{2 c_{k}}\right)$ over $i=1 \ldots I, j=1 \ldots J$ and $k=1 \ldots K$.

The Bayesian update of the probability is iterated many times until the probability converges. Convergence is ascertained by following the log-likelihood function:

$$
\sum_{i=1}^{I} \sum_{j=1}^{J} \log \left(\sum_{k=1}^{K} r_{i j k} s_{i j k}\right) .
$$

The algorithm is terminated when the change in log-likelihood from the previous iteration becomes negligible. Each iteration of the algorithm involves estimating the cluster parameters $\left(h_{k}, v_{k}, c_{k}\right)$, assigning belonging probabilities based on the cluster parameters, checking for convergence, and re-estimating and applying the modulation function. With each iteration, the parameters describing the distributions move towards a better fit and the belonging probabilities change slightly to reflect the new distributions. Finally, the resulting probability map is interpreted as the tissue density [20, 21]. 
From the three probability maps generated (WM, GM and CSF), BRAIM assigns each brain pixel to the tissue class with major probability (Figure 3 ). The SPM package can also be used for skull stripping.

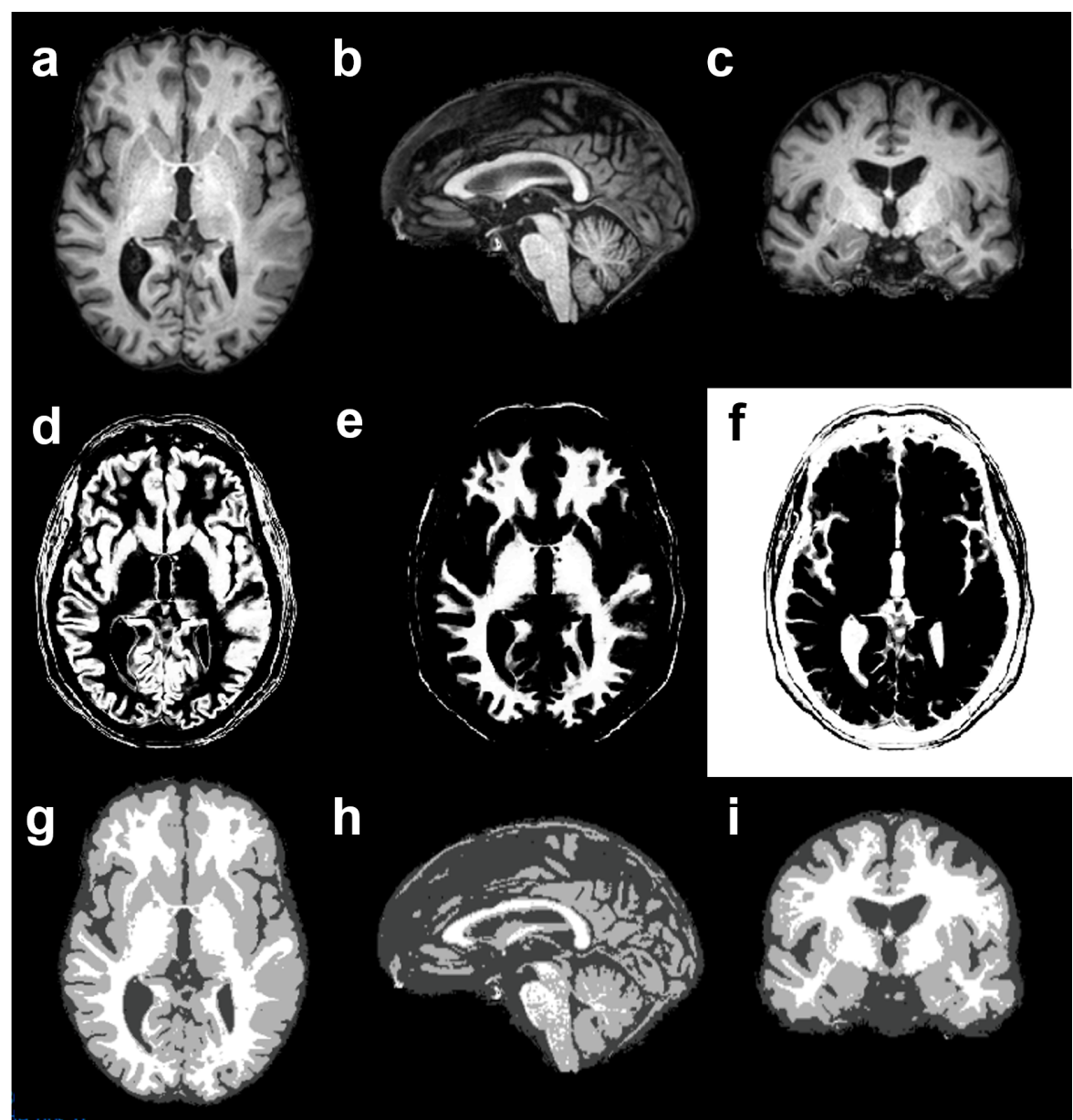

Figure 3: Brain tissue segmentation results. (a-c) MRI study in axial, sagittal and coronal planes, respectively, after skull-stripping by the software. (d-f) Probability maps obtained by gray matter, white matter and cerebrospinal fluid, respectively. (g-i) Segmentation results of white matter, gray matter and cerebrospinal fluid in axial, sagittal and coronal views, respectively. White matter is depicted in white color, gray matter in light gray, and cerebrospinal fluid in dark gray. 


\subsection{Brain structure segmentation}

Basal ganglia structures (putamen, thalamus and caudate nucleus) as well as hippocampus are detected by means of an interactive polygon tool (Figure 4). This tool lets determine the contour of the structures by selecting the vertices of the region of interest. There is no need to segment the structure in each slice since the tool is able to automatically interpolate the contour of the desired structure in non-consecutive slices, so that the segmentation process is considerably facilitated. The contour of the structure of interest must be manually segmented, at least, in the first and in the last slices where it is visible. Afterwards, Fourier descriptors are used to interpolate the existing contours between the manually marked contours [22].

Fourier descriptors, also called discrete Fourier transform (DFT) coefficients, provide a means to represent the boundary of a two-dimensional contour in the frequency domain as follows. Let $x_{k}$ and $y_{k}$ be the coordinates of a K-point 2D contour for $k=\{0,1,2, \ldots, K-1\}$. Each coordinate pair $\left(x_{k}, y_{k}\right)$ can be treated as a complex number so that $s_{k}=x_{k}+j y_{k}$ and the 1D DFT of $s_{k}$ is $a_{u}=\sum_{k=0}^{K-1} s_{k} e^{-j 2 \pi u k / K}$ for $u=\{0,1,2, \ldots, K-1\}$. The coefficients $a_{u}$ are the Fourier descriptors of the boundary. $s_{k}$ can be restored through the inverse Fourier transform of these coefficients as $s_{k}=\frac{1}{K} \sum_{u=0}^{K-1} a_{u} e^{j 2 \pi u k / K}$. If only the first $P$ coefficients are used, an approximation to $s_{k}$ is obtained as $\hat{s}_{k}=\frac{1}{P} \sum_{u=0}^{P-1} a_{u} e^{j 2 \pi u k / K}$. Low-frequency components determine global shape and high-frequency components account for fine detail.

The interpolation between the manually marked contours is based on these concepts. First, the contours previously segmented are resampled to be a power of 2 with two goals: to have the same number of points $K$ in both contours and to make faster the DFT computation. Secondly, the coordinates of the resampled contours are obtained as $s_{1_{k}}=x_{1_{k}}+j y_{1_{k}}$ and $s_{2_{k}}=x_{2_{k}}+j y_{2_{k}}$, respectively. Then, the $1 \mathrm{D}$ DFT of $s_{1_{k}}$ and $s_{2_{k}}$ is computed given $a_{1_{u}}$ and $a_{2_{u}}$. Note that $\{k, u\}=\{0,1,2, \ldots, K-1\}$. Next, the contours of the intermediate slices are lineally predicted in the frequency domain as $a_{i_{u}}=\alpha_{i} a_{1_{u}}+\beta_{i} a_{2_{u}}$, where $i=\{1,2, \ldots, N\}, \alpha_{i}=i / N$ and $\beta_{i}=(N-i) / N$, being $N$ the number of 
intermediate contours to be interpolated. Afterwards, $a_{i_{u}}$ is low-filtered with cutoff at $P=K / 10$, so only $10 \%$ of the total number of descriptors, i.e., the first $P$ descriptors, are taken into account $(u=\{0,1, \ldots, P-1\})$. Finally, the inverse DFT is calculated to convert the intermediate contours to the spatial domain [23].

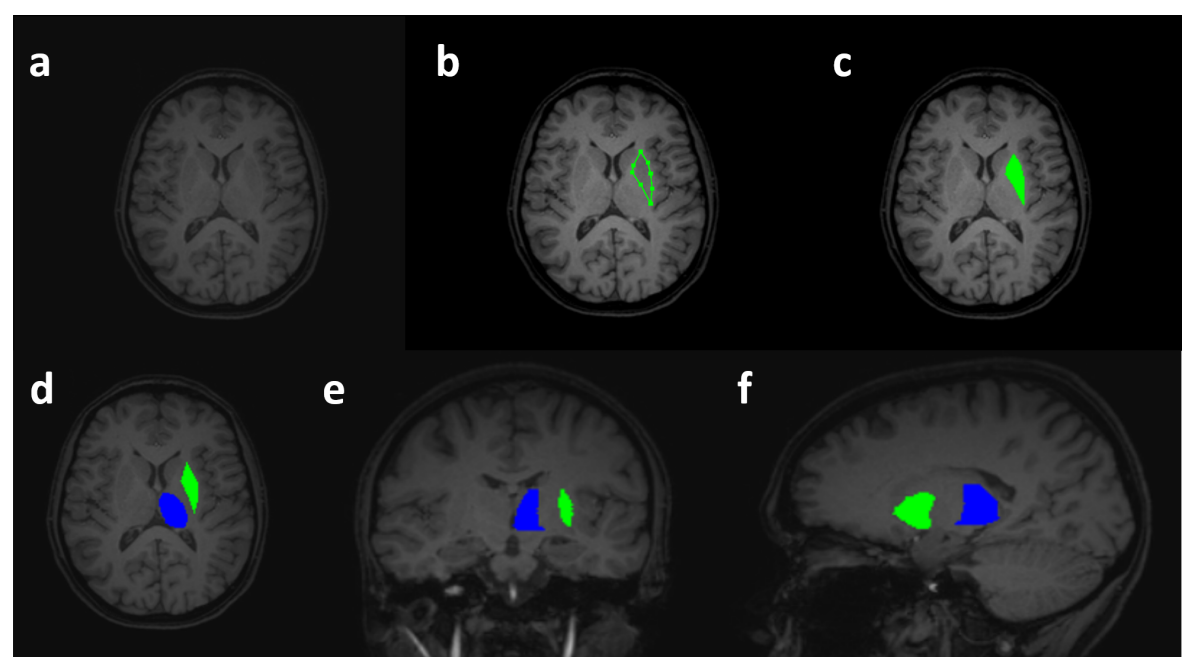

Figure 4: Brain structure segmentation results. (a) Axial T1-wi MRI scan of a healthy 23 year-old female. (b) Interactive polygon tool used for determine the putamen vertices for semi-automatic segmentation. (c) Segmentation results of the putamen in axial plane (green). (d-f) Semi-automatic segmentation results of putamen (green) and thalamus (blue) in axial, coronal and sagittal planes obtained with BRAIM.

\subsection{Brain lesion segmentation}

BRAIM performs a semi-automatic brain lesion segmentation which is based on 3D watershed transformation [24]. Watershed transformation is a segmentation technique for gray-scale images. This algorithm is a powerful segmentation tool whenever the minima of the image represent the objects of interest and the maxima are the separation boundaries between objects. Due to this fact, the input image of this method is usually a gradient image. One problem of this technique is the over-segmentation, which is caused by the existence of numerous 
local minima in the image normally due to the presence of noise. One solution to this problem is using marker-controlled watershed, in which the markers artificially indicate the minima of the image. So, to avoid these deficiencies, BRAIM makes use of the marker-controlled variant. In marker definition not only markers contained inside the object of interest (internal markers) are needed but also a marker that determines the image background (external marker). In particular, BRAIM uses two markers (an internal marker located within the lesion and an external marker outside of the lesion). The user must select a slice where the lesion is clearly visible and choose those markers (only two points), the rest of the lesion segmentation process is completely automatic. The exact location of the seeds has little influence in the final result. The main steps of the method can be observed in Figure 5. The main advantage of this method is that it requires little user interaction. Note that BRAIM is designed to be used in clinical domain and its goal is to make the segmentation process as easier as possible. For example, an algorithm based on active contours could obtain similar results but it would require a more accurate initialization (only two points would not be valid).

The method proposed for lesion segmentation is able to identify different type of lesions independently of their gray intensity level (tumors as meningiomas, glioblastomas, cavernomas... or cystic lesions as pineal cysts, arachnoid cysts...). In Figure 6, an example of the automatic segmentation of a patient diagnosed with brain Glioblastoma is presented.

\subsection{Manual edition tools}

BRAIM also provides additional tools for manual edition of the automatic segmentation results if it is required (pencil tool, paint bucket tool and eraser tool, see Figure 7). If some manual modification is wanted, it must be performed previously to volume computation.

\subsection{Volume computation}

After segmentation, the volume of the lesion and/or the segmented structures can be directly computed and a 3D model of the brain and the lesion generated. 


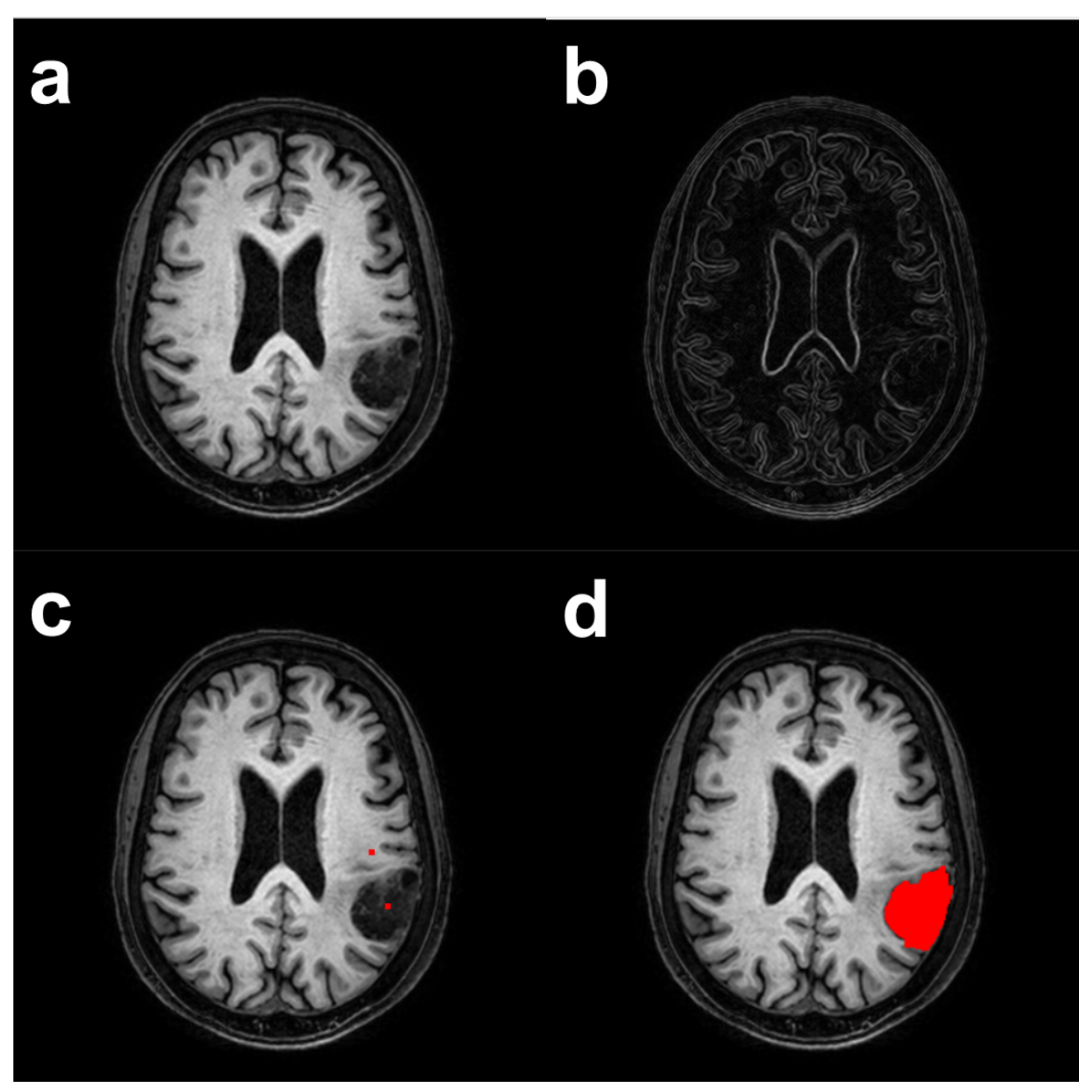

Figure 5: Brain lesion segmentation steps. (a) Original MRI. (b) Gradient image. (c) Markers used in marker-controlled watershed. (d) Final result.

The patient's brain structure volume results are classified with respect to a volumetry control database of healthy subjects that is used as a reference. This comparison allows to better identify brain volume loss, a characteristic feature of neurodegenerative disorders. In the Results section, more information about the control database is provided. In the case of brain lesion segmentation, the volume results obtained can be used in progressive controls for prospective and retrospective tumor analyses. 


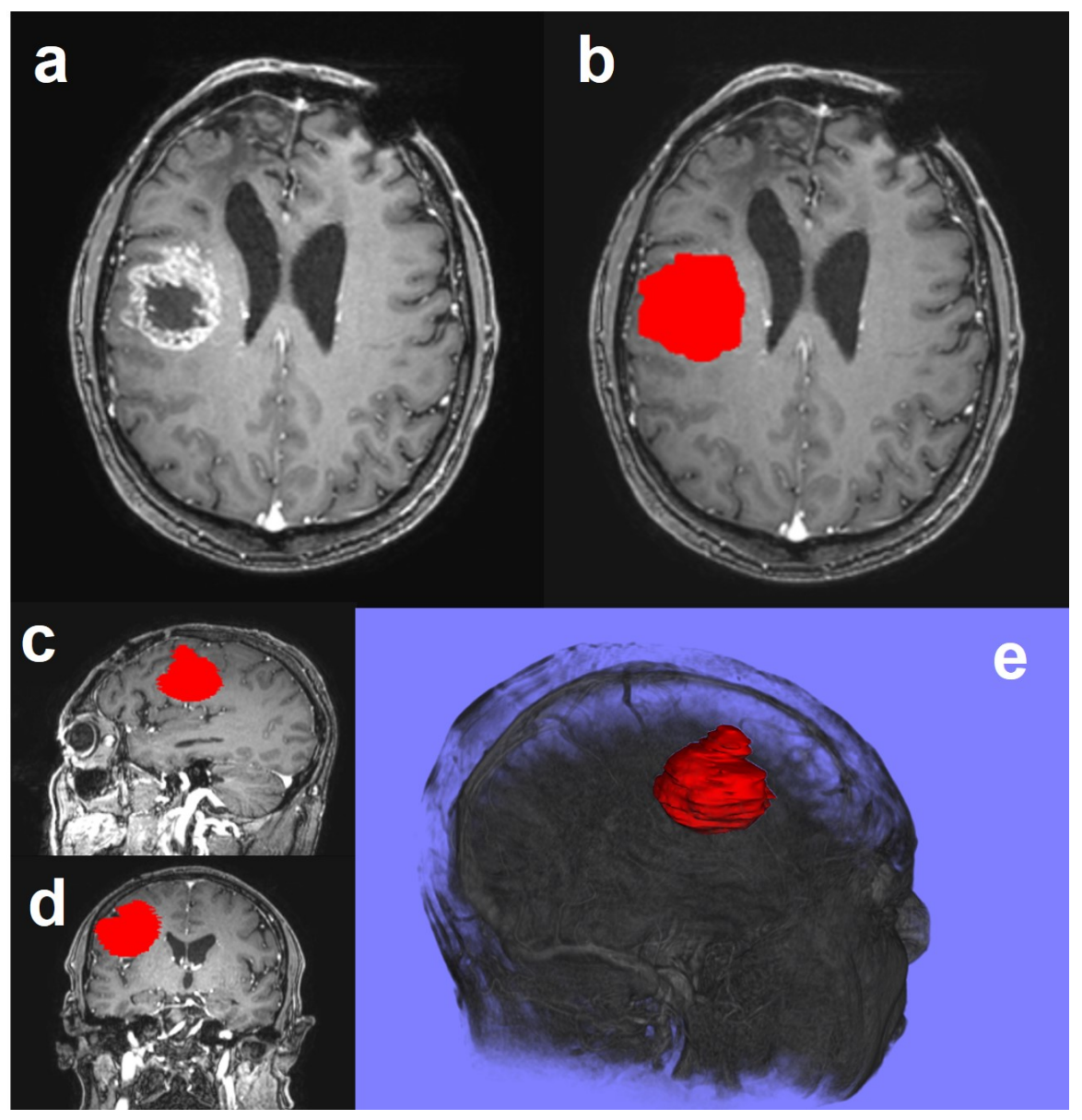

Figure 6: Brain tumor segmentation example in a 53 year-old patient diagnosed with right frontal lobe Glioblastoma. (a) Axial 3D T1-wi after contrast injection (Gadovist, $0.1 \mathrm{mmol} / \mathrm{kg}$ ). (b-d) Segmentation results in axial, coronal and sagittal planes, respectively. (e) $3 \mathrm{D}$ reconstruction of the segmented lesion where it is possible to see the lesion in a better detail. Segmentation results showed a total tumor volume of $34.54 \mathrm{~cm} 3$.

\subsection{Report generator}

The report generator tool creates a predesigned report where the patient's data (name and age), the study date and the volume results are presented (tissue, structure and/or lesion volumes, depending on the study type). It is also possible to show some anatomical images (previously selected by the clinician as 


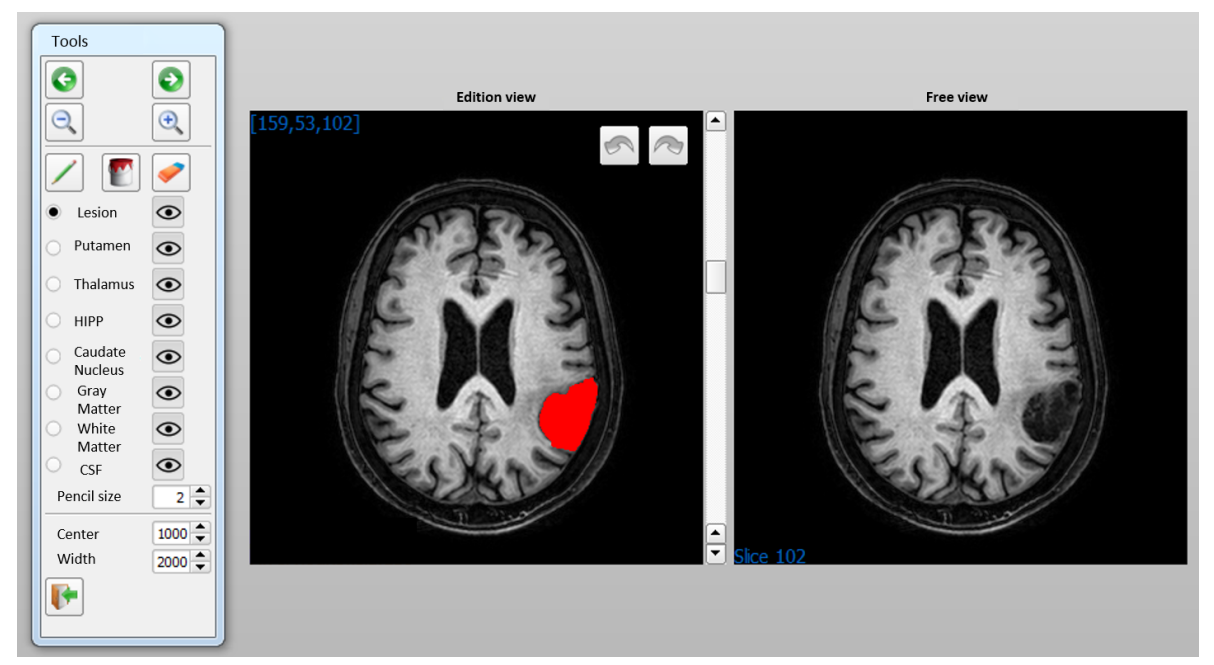

Figure 7: Manual edition tools for segmentation performance and correction provided by BRAIM.

more representative) in axial, sagittal and coronal view and the graphs where brain structure volume results were compared with respect to the volumetry control database.

\section{Results}

\subsection{Validation of the segmentation methods}

For method's performance evaluation, two public databases were used. Both databases provide manually segmented images to be used as ground truth.

The performance of the implemented segmentation methods was evaluated through different concepts in order to quantitatively verify their accuracy. Dice's coefficient (D) describes similarity degree between the ground truth and the segmentation to be evaluated being equal to 1 when segmentation is perfect. Sensitivity (S), also called true positive fraction, measures the proportion of positives that are correctly identified as such; it is established by dividing the correctly classified positive pixels by the total number of positive pixels in the 
gold standard. Positive predictive value (PPV) is the proportion of positive results that are true positive.

\subsubsection{Brain tissue segmentation}

The Internet Brain Segmentation Repository (IBSR, https://www.nitrc. org/projects/ibsr/) and, in particular, the database IBSR V2.0 was used for the validation of brain tissue segmentation. IBSR V2.0 contains the scans of 18 subjects and the corresponding WM and GM manual segmentations.

Table 1 shows the results obtained by the method proposed for tissue segmentation. Both, automatic results which were obtained directly for the segmentation method and the results obtained after a manual modification to correct some misclassification, have been included. Manual modification was performed by an expert neuroradiologist. It can be observed that the automatic results are quite similar to those obtained after manual modification.

Table 1: Results (mean $\pm \mathrm{SD}$ ) obtained by the method proposed for tissue segmentation on IBSR V2.0. Dice's coefficient (D), sensitivity (S) and positive predictive value (PPV). Automatic results and results after manual modification.

\begin{tabular}{|c|c|c|c|c|}
\hline & & $\mathbf{D}$ & $\mathbf{S}$ & PPV \\
\hline \multirow{2}{*}{ 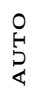 } & Gray matter & $0.781 \pm 0.078$ & $0.736 \pm 0.074$ & $0.836 \pm 0.104$ \\
\hline & White matter & $0.798 \pm 0.121$ & $0.758 \pm 0.174$ & $0.873 \pm 0.069$ \\
\hline \multirow{2}{*}{ 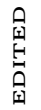 } & Gray matter & $0.781 \pm 0.075$ & $0.751 \pm 0.076$ & $0.823 \pm 0.115$ \\
\hline & White matter & $0.802 \pm 0.121$ & $0.757 \pm 0.174$ & $0.884 \pm 0.068$ \\
\hline
\end{tabular}

\subsubsection{Brain lesion segmentation}

For the validation of brain lesion segmentation, images from BRATS2015 dataset (https://www.smir.ch/BRATS/Start2015) were used. This dataset belongs to Brain Tumor Image Segmentation Challenge [25]. BRATS2015 is divided into two subsets: high-grade and low-grade gliomas, HGG and LGG, respectively. It includes ground truth images which have four different labels: necrosis, edema, non-enhancing tumor and enhancing tumor. The evaluation is 
done for the tumor core region defined as the union of necrosis, non-enhancing tumor and enhancing tumor.

In the same way that in Table 1, Table 2 shows the results obtained by the method proposed for lesion segmentation. Both, automatic results pre and after manual modification, have been included. It can be observed that the proposed method works better on LGG. In that case, manual modification implies a greater improvement than in tissue segmentation. It can be caused by the exiting variability among different lesions. Although, manual modification can be required in some cases, time saving is considerable if it is compared with a complete manual lesion segmentation. In addition to the validation shown in Table 2, a validation based on volume results was also performed. Statistical results did not show any significant differences between the calculated lesion volumes from the automated and edited analyses obtained with BRAIM software and the ground truth results (data not shown for briefness). This result suggested that the obtained lesion volumes with BRAIM software were accurate in all cases.

Table 2: Results (mean $\pm \mathrm{SD}$ ) obtained with the method proposed for lesion segmentation on BRATS2015 images (high-grade and low-grade gliomas, HGG and LGG). Dice's coefficient (D), sensitivity (S) and positive predictive value (PPV). Automatic results and results after manual modification.

\begin{tabular}{|c|c|c|c|c|}
\hline & & D & $\mathbf{S}$ & PPV \\
\hline \multirow[b]{3}{*}{ \& } & HGG & $0.658 \pm 0.111$ & $0.558 \pm 0.175$ & $0.920 \pm 0.180$ \\
\hline & LGG & $0.685 \pm 0.166$ & $0.730 \pm 0.209$ & $0.722 \pm 0.214$ \\
\hline & HGG + LGG & $0.671 \pm 0.138$ & $0.640 \pm 0.207$ & $0.825 \pm 0.218$ \\
\hline & HGG & $0.862 \pm 0.053$ & $0.832 \pm 0.064$ & $0.904 \pm 0.091$ \\
\hline & LGG & $0.800 \pm 0.081$ & $0.800 \pm 0.102$ & $0.820 \pm 0.132$ \\
\hline & HGG + LGG & $0.832 \pm 0.073$ & $0.817 \pm 0.084$ & $0.864 \pm 0.118$ \\
\hline
\end{tabular}




\subsection{Control database of healthy subjects}

The volumetric analyses performed on healthy subjects were used to generate volume normality curves (Figure 8 ). There exists a curve for each type of tissue (WM, GM and CSF) and brain structure (putamen, thalamus, caudate nucleus and hippocampus). The curves represent the maximum and minimum volume values for each age range and can be used to classify a subject as normal or not regarding the volume of their brain tissues and structures. If the volume results of a new subject fall between the curves, as occurs in the example of the Figure 8 , it means that the patient is within the range of normality. If not, tissue and structure volumes will be outside this range and it will indicate that there is a brain volume increase or decrease. In some of the experiments of the following section, this situation will be observed.

Although the initial control database is composed of 50 healthy subjects, BRAIM allows the constant addition of new data so that the database can be infinitely increased. The normality curves are automatically updated when new healthy tissue and structure volumes are included into the control database.

For stablishing a correct database architecture, before incorporating the volume results into the healthy database, a statistical analysis was performed for hippocampus and basal ganglia structures. The objective was to rule out the possibility of interhemispheric volume asymmetries in the healthy population. Statistical results did not show any significant differences in the volume structures between hemispheres (data not shown for briefness) and therefore total volume of both structures was included in the data base as a whole (i.e. total volume of both thalamus, both caudate nucleus...).

In the selected control participants, brain volume values obtained with BRAIM CAD system were within the range of normality as described previously in the literature $[26,27,28]$. The comparative study between sexes showed that males presented significant higher brain volumes compared to females, reflecting sexual dimorphism as described previously [29, 30] (see Table 3). Results also showed the presence of a progressive brain volume loss (as observed in the normality curves) with age as reported previously [31] (see Figure 9). 

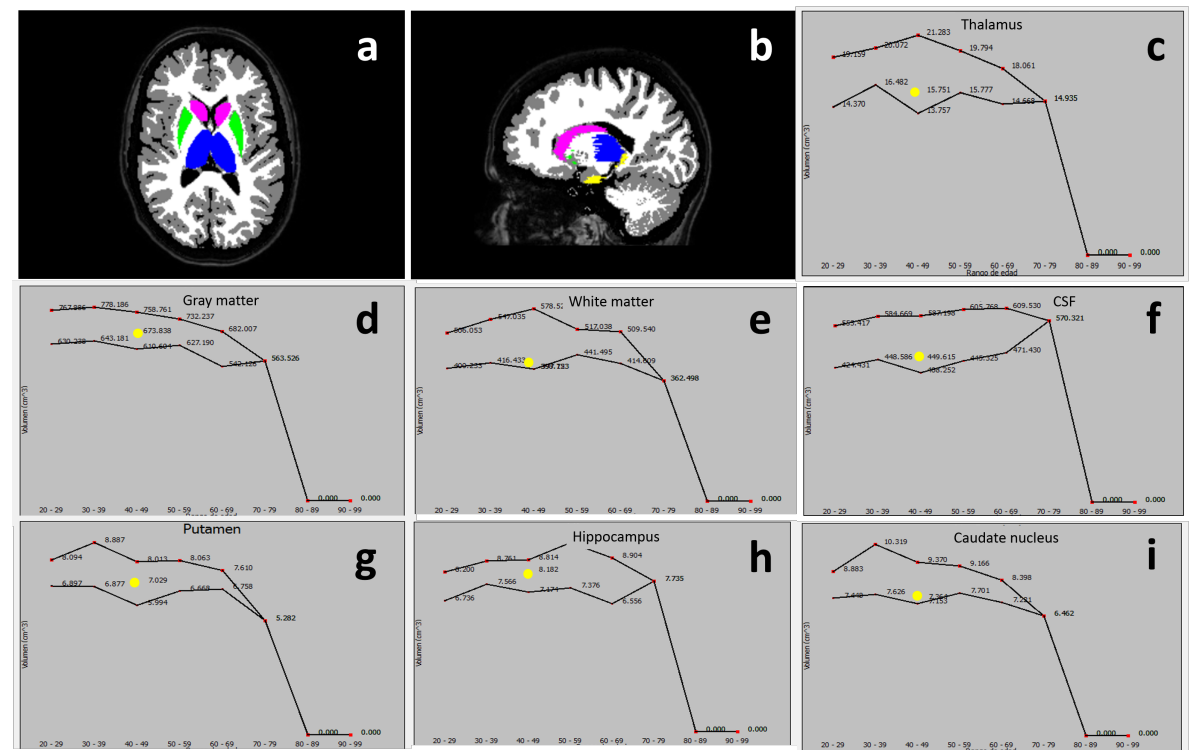

Figure 8: Volume results obtained in a 40 year-old healthy female. (a-b) Representation of the segmented structures in axial and sagittal planes, respectively. (c-i) Graphs presenting the subject classification within the healthy control database ( $\mathrm{x}$-axis age, $\mathrm{y}$-axis volume) of the different structures analyzed. Subject's results are displayed as a big yellow dot. Small red dots represent the maximum and minimum volume values of the normality range for each age group. Results showed that the subject was within the normality range when compared to the control database.

Table 3: Volume results obtained in the control group. Results are expressed as mean \pm SD. $\mathrm{p}$ values obtained with a t-student test are shown.

\begin{tabular}{lccc}
\hline & Females $(\mathbf{n = 2 5})$ & Males $(\mathbf{n = 2 5})$ & $\mathbf{p}$ \\
\hline Total brain volume & $1478.96 \pm 152.77$ & $1625.34 \pm 144.44$ & 0.006 \\
Gray matter & $759.25 \pm 67.77$ & $830.94 \pm 75.85$ & 0.006 \\
White matter & $409.05 \pm 58.88$ & $452.77 \pm 62.4$ & 0.041 \\
CSF & $310.64 \pm 82.86$ & $341.62 \pm 62.69$ & 0.22 \\
\hline
\end{tabular}

\subsection{Experiments using BRAIM}

In order to evaluate the software performance in some patients and demonstrate its usefulness, four patients with diagnosed pathologies were selected. 


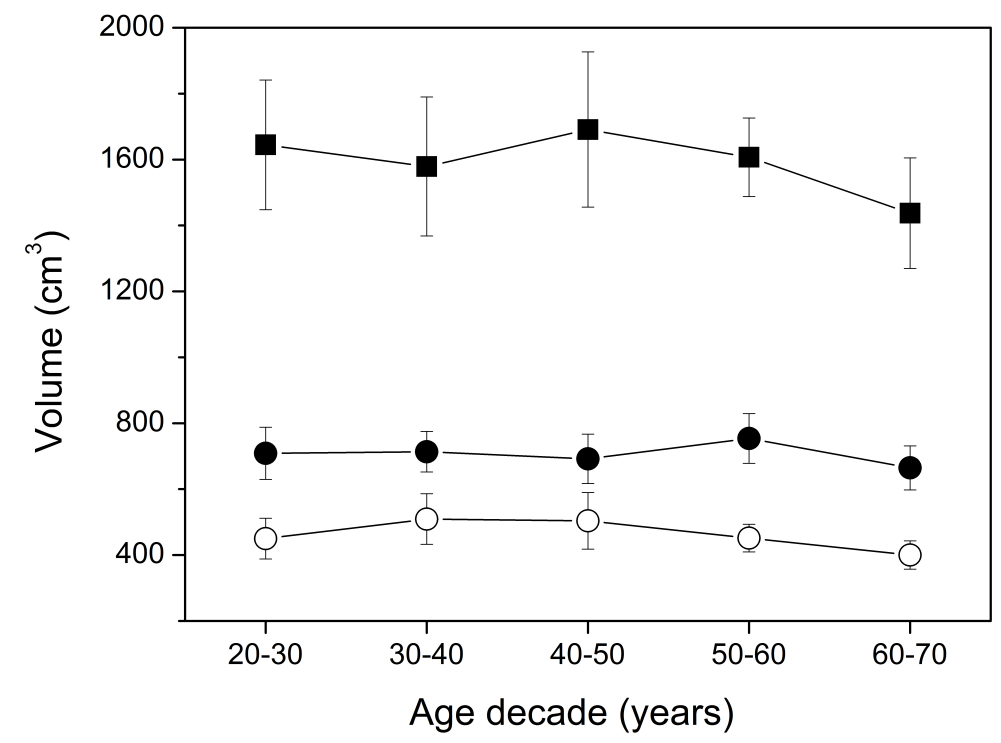

Figure 9: Volume results obtained in the control group with BRAIM software. Values are expressed as mean standard deviation. (ם) Total brain volume, (•) gray matter volume, (o) white matter volume.

The brain tissues, structures and/or lesions of these patients were segmented and their volume calculated making use of the tools provided in BRAIM.

\subsubsection{Experiment 1: brain tissue and structure volumes}

The brain tissue and structure volumes of two patients diagnosed with Multiple Sclerosis were analyzed (Patients A and B). Patient A presented cognitive tests suggesting mild cognitive impairment whereas Patient B presented normal cognitive test results (see Materials and Methods for more information). No significant differences were observed with treatment, time of diagnosis, age and sex. However brain volume results, when they were compared to the healthy control database, showed brain volume loss in different brain areas that was more significant in Patient A. Patient A also presented significant volume loss associated to hippocampus, a brain structure related to short-term and long-term memory 
and spatial memory and navigation $[32,33]$. Therefore, the results obtained with BRAIM allowed us to detect volume loss, the specific areas affected and to correlate them with the patient symptoms (see Figures 10 and 11).

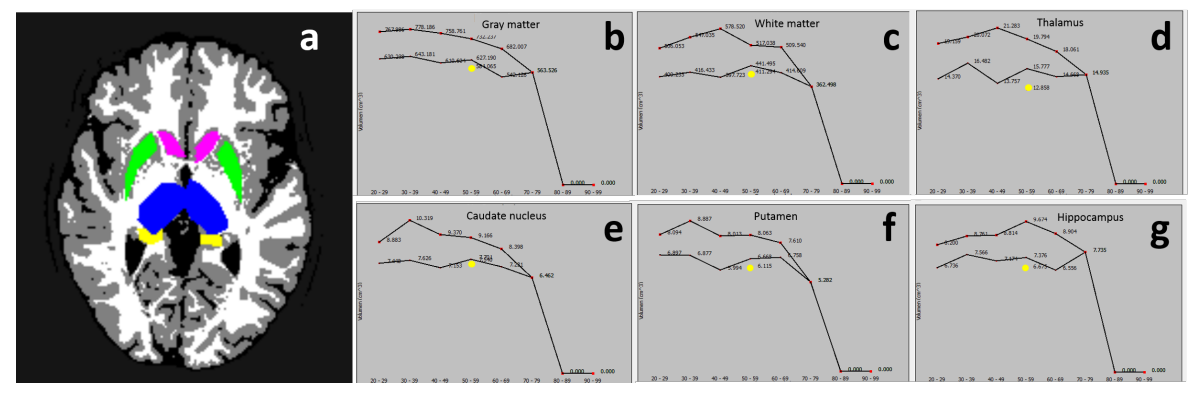

Figure 10: Volume results obtained in a 47 year-old female patient diagnosed with Multiple Sclerosis subtype remitent-recidivant for eleven years (Patient A). (a) Segmented structures in axial plane. (b-g) Graphs representing the subject classification in the healthy control database (x-axis age, y-axis volume) of the different structures analyzed. Subject's results (big dots in yellow) showed the presence of significant volume loss affecting white and gray matter, thalamus, hippocampus, putamen and caudate nucleus.
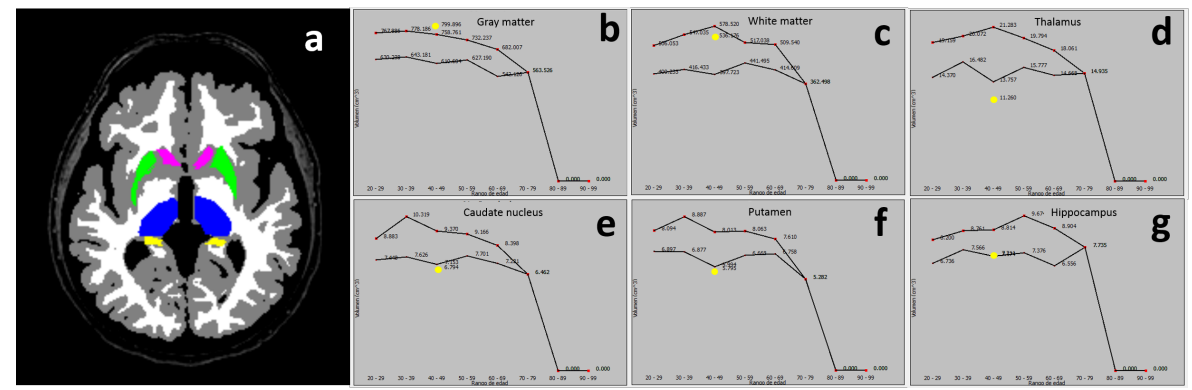

Figure 11: Volume results obtained in a 48 year-old female patient diagnosed with Multiple Sclerosis subtype remitent-recidivant for fourteen years (Patient B). (a) Segmented structures in axial plane. (b-g) Graphs representing the subject classification in the healthy control database (x-axis age, y-axis volume) of the different structures analyzed. Subject's segmentation results (big dots in yellow) only showed the presence of significant volume loss affecting thalamus, putamen and caudate nucleus. 


\subsubsection{Experiment 2: brain lesion volumes}

The lesion volume of other two patients diagnosed with brain tumors (Patients $\mathrm{C}$ and D) were also analyzed with the presented CAD system. Lesion segmentation with BRAIM software allowed us to obtain a robust and precise measurement of the lesion burden in the patients. Segmentation was performed automatically with BRAIM and manually corrected in case necessary by an experienced researcher (A.B-S).

Figure 12 displays the volume results of a 25 year-old female patient diagnosed with a pontine tumor. Because of the lesion location surgery was avoided and an experimental treatment with Chemotherapy was applied. MRI was performed to better control the treatment efficacy. As it is shown in the graph, the treatment let a significant tumor reduction, which was related to a clinical improvement (diplopia), being the patient at the moment clinically stable. These results allowed us to precisely calculate the tumor reduction after treatment and to better monitor the treatment efficacy.

Figure 13 shows the segmentation results obtained in a 27 year-old male patient diagnosed with an astrocitoma III. The tumor affected both frontal lobes and corpus callosum. Complete tumor surgery was not possible because of the lesion location and extension. After definite diagnose with histopathology, Radiotherapy and Chemotherapy were administrated to the patient. The graph shows the lesion evolution with time and treatment. As it can be seen, the lesion was significantly reduced with treatment, however after last surgery (month 27) there was a worsening of the patient symptoms with a significant lesion volume increase (month 43). Histopathology results showed that the lesion had transformed to a Glioblastoma (grade IV tumor).

\section{Discussion}

As mentioned before, BRAIM is able to segment brain tissues, structures and/or lesions in addition to perform volumetric analyses. One of the main advantages of the software is that it is easy to use and could be operated in a 


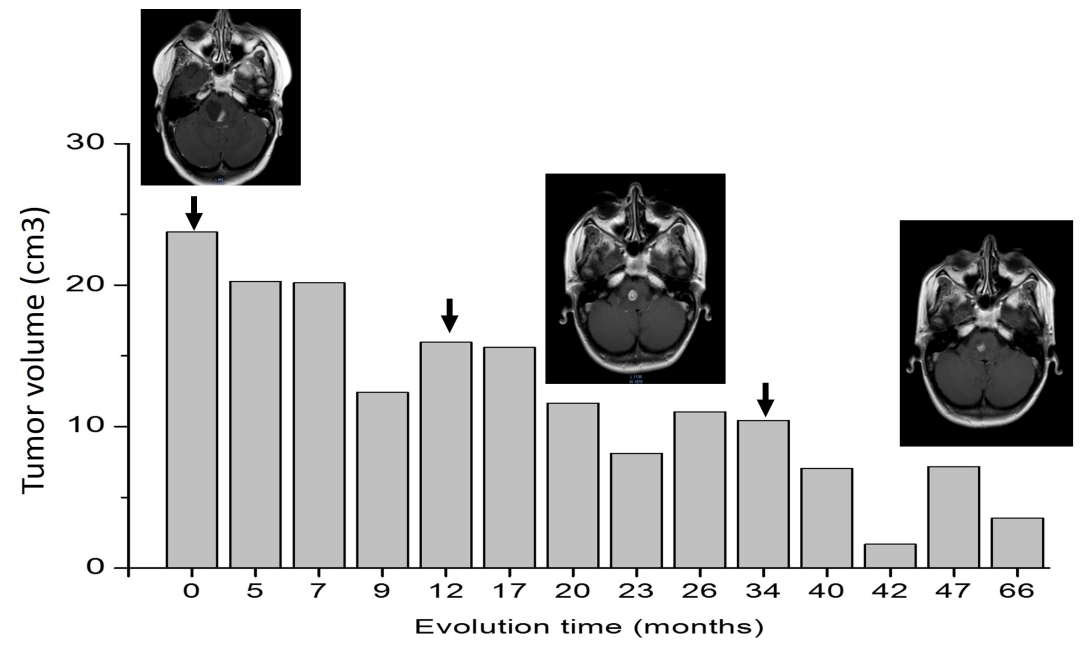

Figure 12: Lesion volume results obtained with BRAIM CAD system in a 25 year-old female diagnosed with a pontine tumor (Patient C). Biopsy was not performed because of the lesion location. Chemotherapy was applied in order to control tumor growth. MRI scans were performed for lesion control and treatment efficacy monitoring. MR images on the graphs (axial T1-wi after contrast injection) display the lesion at the beginning of the treatment $(23.74 \mathrm{~cm} 3)$, the lesion after two sessions of chemotherapy $(11.66 \mathrm{~cm} 3)$ and the last control performed after three chemotherapy sessions $(3.52 \mathrm{~cm} 3)$. Chemotherapy treatments are shown in the graph as arrows.

common PC with a Windows operating system. Comparing to other similar programs e.g. SPM, FSL, FreeSurfer (available through fil.ion.ucl.ac.uk/spm/ software/spm12; fsl.fmrib.ox.ac.uk/fsldownloads/; freesurfer.net/; respectively) its use do not require the installation of additional software, MAT$\mathrm{LAB}$, virtual machines or programming knowledge, which makes easier its use in the clinical setting. Segmentations are performed in a very intuitive an easy way, and the results are robust and reproducible. In particular, little user interaction is needed for lesion and structure segmentation. In the case of lesion segmentation, the interaction is reduced to the only selection of two points, a point within the lesion and other outside, as it was explained in Subsection 3.4. Structure segmentation requires a bit more interaction, the user must determine 


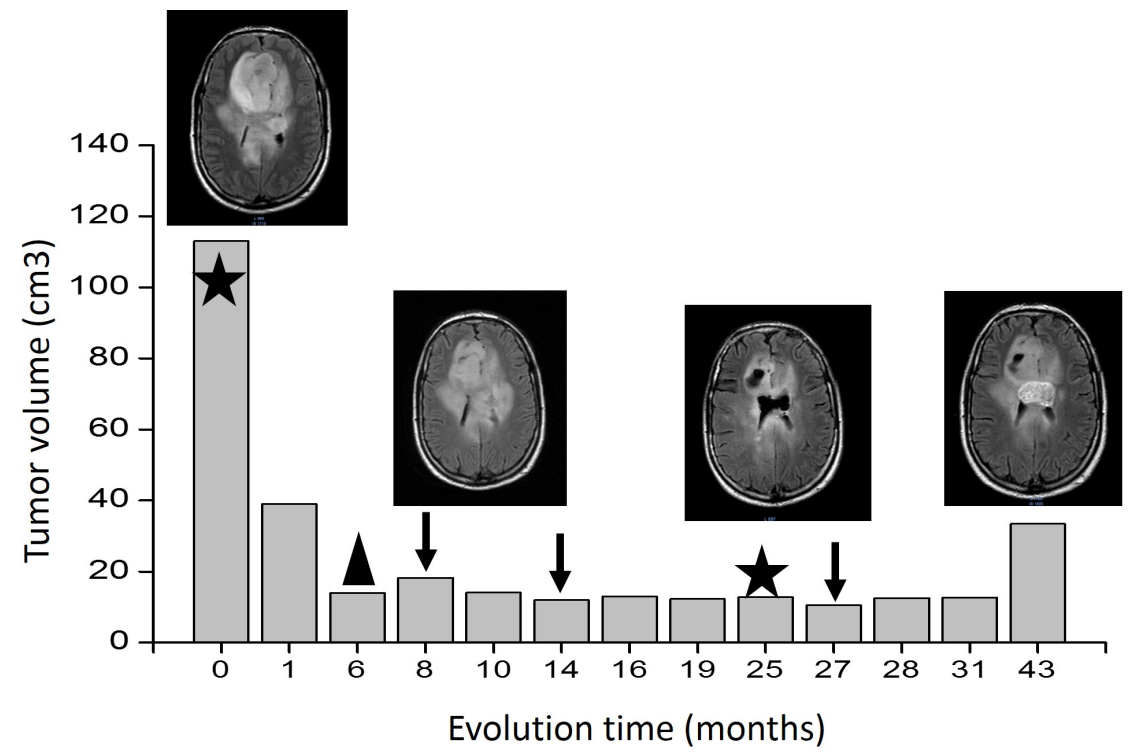

Figure 13: Lesion volume results obtained with BRAIM software in a 27 year-old male diagnosed with astrocitoma III after biopsy (Patient D). The lesion was affecting both frontal lobes and corpus callosum. Initial volume was $113 \mathrm{~cm}^{3}$, after partial surgery the lesion volume was of $39 \mathrm{~cm}^{3}$, suggesting that the $65 \%$ of the lesion was removed. Chemotherapy (CHT) and Radiotherapy (RT) were applied in order to control the rest tumor growth. MRI scans were performed for lesion control and treatment efficacy monitoring. MR images on the graphs (axial FLAIR for better visualization of the lesion extension because the lesion did not present contrast enhancement) display the initial lesion, the lesion after two sessions of CHT and one session of RT $\left(14.1 \mathrm{~cm}^{3}\right)$ and the last control performed after a second surgery and a chemotherapy session $\left(33.4 \mathrm{~cm}^{3}\right)$. Chemotherapy treatments are shown in the graph as arrows, surgery is shown as a star and RT is shown as a triangle.

by means of mouse clicks the vertices of the structure of interest, at least, in the first and in the last slice of the MRI volume where the structure is visible (see Subsection 3.3 for more details). In addition, the results of BRAIM could be always manually corrected in case they are not satisfactory through edition tools such as pencil tool, paint bucket and eraser tool. This is a very important feature of the software and constitutes a main advantage respect to other software since it avoids that some patients could not be included in the study 
because of problems with the segmentation algorithms. Thus, BRAIM allows to a more efficient use of the data and prevents data loss.

The software performance was tested and the system was used to generate a volumetry control database. Even with only a database of 50 healthy subjects, the analysis preformed in patients diagnosed with neurodegenerative disorders allowed us to detect brain volume loss as well as local differences (related to brain structures) that were related to clinical symptoms. In addition, the system was able to classify the patient within the database providing objective results. In oncology patients, the results presented a good correlation with the treatment application and patient evolution; allowing us to better predict the outcome.

The presence of an internal control database is extremely useful as it allows to detect brain volume changes in the patients more efficiently. To our knowledge there is not at the moment any other software available that allows a direct patient classification within an internal control database. In this context, it is important to note that the software is able to compare and classify different brain areas. The results are shown in a simple and intuitive graph system allowing a fast classification of the patients between normal or pathological. Moreover, the ability to detect volume loss in specific brain areas is also of value, as it has been shown that different neurodegenerative diseases present different neurodegenerative patterns $[34,35,36]$. Therefore, BRAIM results could be used in the clinical setting as a complementary tool to more precisely diagnose brain volume loss or confirm a diagnosis when there is a clinical suspicion of a specific disease.

Overall, BRAIM software allowed us to obtain in a fast and simple way, information that is not currently available by conventional MRI clinical procedures. According to our preliminary results, we believe that the information provided by the software may be extremely useful in the clinical setting for a better patient management and treatment monitoring. 


\section{Conclusions}

In this paper, BRAIM, a new CAD system for brain pathologies diagnosis and monitoring was presented. The software is featured with different userfriendly tools to help clinicians in brain segmentation and volume computation. Specifically, BRAIM allows to perform segmentation and volume measurement of white, gray matter and cerebrospinal fluid; basal ganglia structures and hippocampus; as well as brain lesions. Tissue and lesion segmentations are completely automatic although they can be manually corrected if needed. Brain structures segmentations are semi-automatic and need that an expert select the vertices of the structures in their initial and final slice.

BRAIM software presents numerous advantages for clinicians, as it is fast, robust and easy to work with. From this perspective, it is expected its progressive incorporation in the clinical setting as a tool to help diagnosis and optimal patient monitoring.

In future work, a larger test of the application on more pathological patients will be done. The volumetry control database will also be continually increased. Finally, exhaustive work will be performed for brain structure segmentation by means of completely automatic methods.

\section{Acknowledgements}

This work has been supported by the Centro para el Desarrollo Tecnológico Industrial (CDTI) under the project BRAIM (IDI-20130020).

The authors also would like to thank the following people:

Our subjects for their time and help.

Our MR technologists Mr. David González García, Miss. Patricia Ibáñez Lucena, and specially Miss. Mayra Sánchez Cillero for their outstanding technical support during the acquisition of the studies.

The MR brain data sets and their manual segmentations used for brain tissue validation were provided by the Center for Morphometric Analysis at 
Massachusetts General Hospital and are available at http://www.cma.mgh. harvard.edu/ibsr/.

Brain tumor image data used in this article were obtained from the MICCAI 2013 Challenge on Multimodal Brain Tumor Segmentation. The challenge database contain fully anonymized images from the Cancer Imaging Archive.

\section{Appendix A. Main methods of BRAIM}

Table A.4 enumerates the main methods included in BRAIM along with the main functions or classes used in each method and their corresponding libraries. Note that for the development of the methods that make use of the ITK, VTK and Qt libraries, the mentioned functions were invoked from own code and combined with other own algorithms.

\section{References}

[1] A. Shaqiri, A. Clarke, M. Kunchulia, D. Herzig, K. Pilz, M. H. Herzog, The effects of aging on perception and cognition, in: Journal of Vision, Vol. 15, Association for Research in Vision and Ophthalmology, 2015, p. 802.

[2] B. Shatenstein, P. Barberger-Gateau, Prevention of age-related cognitive decline: Which strategies, when, and for whom?, Journal of Alzheimer's Disease (48) (2015) 35-53.

[3] M. C. Kenfield, D. B. Arciniegas, C. A. Anderson, K. L. Howard, C. M. Filley, When cognitive evaluation does not disclose a neurologic disorder: Experience of a university behavioral neurology clinic:, Cognitive and Behavioral Neurology 23 (2010) 112-118.

[4] D. S. Knopman, The initial recognition and diagnosis of dementia, The American Journal of Medicine 104 (4) (1998) 2S-12S.

[5] R. J. Schindler, Dementia with cerebrovascular disease: the benefits of early treatment, European Journal of Neurology 12 (2005) 17-21. 
Table A.4: Main methods of BRAIM used for interface development, tissue-structure-lesion segmentation and volume computation: general description, main function or class and corresponding library .

\begin{tabular}{|c|c|c|c|}
\hline & Description & Main function/class & Library \\
\hline \multirow{7}{*}{ 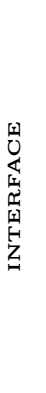 } & Open directory & QFileDialog::getExistingDirectory & Qt \\
\hline & Select MRI series & QInputDialog::getItem & Qt \\
\hline & Read MRI series & itk::ImageSeriesReader & ITK \\
\hline & Contrast enhancement & Own function & - \\
\hline & Manual edition & QDialog, QFrame & Qt \\
\hline & Save results & itk::ImageFileWriter & ITK \\
\hline & Report generation & QPrinter & Qt \\
\hline \multirow{3}{*}{ 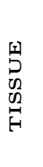 } & Dicom to Nifti conversion & itk::NiftiImageIO & ITK \\
\hline & WM, GM, CSF segmentation & spm_preproc_run & $\mathrm{SPM}$ \\
\hline & Tissue combination & Own function & - \\
\hline \multirow{2}{*}{ 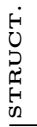 } & Contour segmentation & QDialog, QFrame & Qt \\
\hline & Contour interpolation & Own function & - \\
\hline \multirow{2}{*}{ 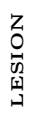 } & Increasing weighted average filter & Own function & - \\
\hline & Marker-controlled watershed & Own function & - \\
\hline \multirow{5}{*}{$\begin{array}{l}\sum_{1} \\
\sum_{j} \\
0 \\
0 \\
>\end{array}$} & Volume computation & Own function & - \\
\hline & ITK to VTK conversion & itk::ImageToVTKImageFilter & ITK \\
\hline & 3D model generation & vtkImageMarchingCubes & VTK \\
\hline & $3 \mathrm{D}$ model representation & vtkActor & VTK \\
\hline & Volume comparison & QPixmap, QPainter & Qt \\
\hline
\end{tabular}

[6] A. Fjell, L. Westlye, H. Grydeland, I. Amlien, T. Espeseth, I. Reinvang, N. Raz, A. Dale, K. Walhovd, Accelerating cortical thinning: Unique to dementia or universal in aging?, Cerebral Cortex 24 (4) (2014) 919-934.

[7] C. R. Jack, M. M. Shiung, S. D. Weigand, P. C. O’Brien, J. L. Gunter, B. F. Boeve, D. S. Knopman, G. E. Smith, R. J. Ivnik, E. G. Tangalos, R. C. Petersen, Brain atrophy rates predict subsequent clinical conversion 
in normal elderly and amnestic mci, Neurology 65 (8) (2005) 1227-1231.

[8] J. Jiang, P. Sachdev, D. M. Lipnicki, H. Zhang, T. Liu, W. Zhu, C. Suo, L. Zhuang, J. Crawford, S. Reppermund, et al., A longitudinal study of brain atrophy over two years in community-dwelling older individuals, NeuroImage 86 (2014) 203-211.

[9] K. M. Pohl, E. Konukoglu, S. Novellas, N. Ayache, A. Fedorov, I.-F. Talos, A. Golby, W. M. Wells, R. Kikinis, P. M. Black, A new metric for detecting change in slowly evolving brain tumors: validation in meningioma patients, Neurosurgery 68 (1 Suppl OPERATIVE) (2011) 225.

[10] D. Chow, J. Qi, X. Guo, V. Miloushev, F. Iwamoto, J. Bruce, A. Lassman, L. Schwartz, A. Lignelli, B. Zhao, et al., Semiautomated volumetric measurement on postcontrast mr imaging for analysis of recurrent and residual disease in glioblastoma multiforme, American Journal of Neuroradiology 35 (3) (2014) 498-503.

[11] M. Vaidyanathan, L. Clarke, L. Hall, C. Heidtman, R. Velthuizen, K. Gosche, S. Phuphanich, H. Wagner, H. Greenberg, M. Silbiger, Monitoring brain tumor response to therapy using mri segmentation, Magnetic resonance imaging 15 (3) (1997) 323-334.

[12] A. W.-C. Liew, H. Yan, Current methods in the automatic tissue segmentation of 3d magnetic resonance brain images, Current Medical Imaging Reviews 2 (1) (2006) 91-103.

[13] D. L. Pham, C. Xu, J. L. Prince, Current methods in medical image segmentation 1, Annual review of biomedical engineering 2 (1) (2000) 315-337.

[14] L. K. Sheridan, H. E. Fitzgerald, K. M. Adams, J. T. Nigg, M. M. Martel, L. I. Puttler, M. M. Wong, R. A. Zucker, Normative symbol digit modalities test performance in a community-based sample, Archives of Clinical Neuropsychology 21 (1) (2006) 23-28. 
[15] L. Rami, J. Molinuevo, R. Sanchez-Valle, B. Bosch, A. Villar, Screening for amnestic mild cognitive impairment and early alzheimer's disease with m@t (memory alteration test) in the primary care population, International journal of geriatric psychiatry 22 (4) (2007) 294-304.

[16] H. M. González, K. E. Whitfield, B. T. West, D. R. Williams, P. A. Lichtenberg, J. S. Jackson, Modified-symbol digit modalities test for african americans, caribbean black americans, and non-latino whites: Nationally representative normative data from the national survey of american life, Archives of Clinical Neuropsychology 22 (5) (2007) $605-613$.

[17] A. Drake, B. Weinstock-Guttman, S. Morrow, D. Hojnacki, F. Munschauer, R. Benedict, Psychometrics and normative data for the multiple sclerosis functional composite: replacing the pasat with the symbol digit modalities test, Multiple Sclerosis Journal 16 (2) (2010) 228-237.

[18] L. Rami, B. Bosch, R. Sanchez-Valle, J. Molinuevo, The memory alteration test (m@t) discriminates between subjective memory complaints, mild cognitive impairment and alzheimer's disease, Archives of Gerontology and Geriatrics 50 (2) (2010) $171-174$.

[19] N. Custodio, D. Lira, E. Herrera-Perez, L. Nuñez del Prado, J. Parodi, E. Guevara-Silva, S. Castro-Suarez, R. Montesinos, P. Cortijo, The memory alteration test discriminates between cognitively healthy status, mild cognitive impairment and alzheimer's disease, Dement Geriatr Cogn Disord Extra 4 (2014) 314-321.

[20] J. Ashburner, K. Friston, Image segmentation, in: R. Frackowiak, K. Friston, C. Frith, R. Dolan, K. Friston, C. Price, S. Zeki, J. Ashburner, W. Penny (Eds.), Human Brain Function, 2nd Edition, Academic Press, 2003.

[21] M. K. Chung, Statistical and computational methods in brain image analysis, CRC Press, 2014. 
[22] C. T. Zahn, R. Z. Roskies, Fourier descriptors for plane closed curves, IEEE Transactions on Computers C-21 (3) (1972) 269-281.

[23] F. Marques, B. Llorens, A. Gasull, Prediction of image partitions using fourier descriptors: application to segmentation-based coding schemes, IEEE Transactions on Image Processing 7 (4) (1998) 529-542.

[24] S. Beucher, F. Meyer, The morphological approach to segmentation: The watershed transformation, in: Mathematical Morphology in Image Processing, E. R. Dougherty, 1992, Ch. 12, p. $433 ? 481$.

[25] B. Menze, A. Jakab, S. Bauer, J. Kalpathy-Cramer, K. Farahani, J. Kirby, Y. Burren, N. Porz, J. Slotboom, R. Wiest, L. Lanczi, E. Gerstner, et al., The Multimodal Brain Tumor Image Segmentation Benchmark (BRATS), IEEE Transactions on Medical Imaging (2014) 33.

[26] Y. Ge, R. I. Grossman, J. S. Babb, M. L. Rabin, L. J. Mannon, D. L. Kolson, Age-related total gray matter and white matter changes in normal adult brain. part i: volumetric mr imaging analysis, American Journal of Neuroradiology 23 (8) (2002) 1327-1333.

[27] F. Klauschen, A. Goldman, V. Barra, A. Meyer-Lindenberg, A. Lundervold, Evaluation of automated brain mr image segmentation and volumetry methods, Human Brain Mapping 30 (4) (2009) 1310-1327.

[28] C. Á. Szabó, J. L. Lancaster, J. Xiong, C. Cook, P. Fox, Mr imaging volumetry of subcortical structures and cerebellar hemispheres in normal persons, American Journal of Neuroradiology 24 (4) (2003) 644-647.

[29] J. S. Allen, H. Damasio, T. J. Grabowski, J. Bruss, W. Zhang, Sexual dimorphism and asymmetries in the gray-white composition of the human cerebrum, Neuroimage 18 (4) (2003) 880-894.

[30] H. Lemaître, F. Crivello, B. Grassiot, A. Alpérovitch, C. Tzourio, B. Mazoyer, Age-and sex-related effects on the neuroanatomy of healthy elderly, Neuroimage 26 (3) (2005) 900-911. 
[31] C. D. Smith, H. Chebrolu, D. R. Wekstein, F. A. Schmitt, W. R. Markesbery, Age and gender effects on human brain anatomy: a voxel-based morphometric study in healthy elderly, Neurobiology of aging 28 (7) (2007) $1075-1087$.

[32] R. J. Sutherland, M. P. Weisend, D. Mumby, R. S. Astur, F. M. Hanlon, A. Koerner, M. J. Thomas, Y. Wu, S. N. Moses, C. Cole, et al., Retrograde amnesia after hippocampal damage: recent vs. remote memories in two tasks, Hippocampus 11 (1) (2001) 27-42.

[33] E. A. Maguire, D. G. Gadian, I. S. Johnsrude, C. D. Good, J. Ashburner, R. S. Frackowiak, C. D. Frith, Navigation-related structural change in the hippocampi of taxi drivers, Proceedings of the National Academy of Sciences 97 (8) (2000) 4398-4403.

[34] H. Wolf, V. Jelic, H.-J. Gertz, A. Nordberg, P. Julin, L.-O. Wahlund, A critical discussion of the role of neuroimaging in mild cognitive impairment*, Acta Neurologica Scandinavica 107 (s179) (2003) 52-76.

[35] L. Serra, M. Cercignani, D. Lenzi, R. Perri, L. Fadda, C. Caltagirone, E. Macaluso, M. Bozzali, Grey and white matter changes at different stages of alzheimer's disease, Journal of Alzheimer's Disease 19 (1) (2010) 147159.

[36] R. Barber, I. McKeith, C. Ballard, J. O'Brien, Volumetric mri study of the caudate nucleus in patients with dementia with lewy bodies, alzheimer's disease, and vascular dementia, Journal of Neurology, Neurosurgery \& Psychiatry 72 (3) (2002) 406-407. 